\title{
Efectividad de la mezcla antibiótica triclaritro en tratamientos pulpares de dientes deciduos
}

\section{Effectiveness of the triclaritro antibiotic mixture in pulp treatments for deciduous teeth}

Aarón Josué Ramos Ramos ${ }^{1 \mathrm{a}}$, Jaime Barcena Taco ${ }^{2 b}$

iD https://orcid.org/0000-0003-2288-337X

iD https://orcid.org/0000-0003-3082-3369

${ }^{1}$ Investigador independiente.

${ }^{2}$ Escuela Profesional de Odontología, Universidad Nacional Jorge Basadre Grohmann. Tacna, Perú. Asociación de Periodoncia y Osteointegración, Miembro de la Sociedad de Prótesis Dental y Maxilofacial.

${ }^{a}$ Cirujano Dentista.

${ }^{b}$ Docente, Especialista en Periodoncia e Implantología, Magister en Docencia Universitaria y Gestión Educativa.

\begin{abstract}
Resumen
Objetivo: Evaluar la efectividad de la mezcla antibiótica triclaritro en la remisión de signos y síntomas, antes y después del tratamiento pulpar de dientes deciduos con diagnóstico de absceso apical agudo de niños de 4 a 7 años que acudieron a la clínica odontológica de la Universidad Nacional Jorge Basadre Grohmann (Tacna, 2017). Metodología: Estudio de tipo descriptivo, de corte longitudinal prospectivo. Población de estudio: 23 niños de 4 a 7 años de edad de ambos sexos, a quienes se les diagnosticó necrosis pulpar; la información obtenida se registró en una ficha de recolección de datos. Resultados: Se observó una diferencia significativa favorable entre los signos y síntomas clínicos al inicio y al final, después de la aplicación de la mezcla antibiótica triclaritro durante los periodos experimentales: 7 días y 15 días. Conclusión: Se llegó a la conclusión que la mezcla antibiótica triclaritro es efectiva en el $100 \%$ de los casos en la remisión de signos y síntomas, después del tratamiento pulpar de dientes deciduos diagnosticados con absceso apical agudo de niños de 4 a 7 años que acudieron a la clínica odontológica de la Universidad Nacional Jorge Basadre Grohmann.
\end{abstract}

Palabras clave: Endodoncia, pulpectomía, diente deciduo, antibióticos.

\begin{abstract}
Aim: To evaluate the effectiveness of the triclaritro antibiotic mixture in pulp treatments of deciduous teeth of children from 4 to 7 years old who attended the dental clinic of the Jorge Basadre Grohmann National University. (Tacna, 2017). Methodology: Study of descriptive type, of prospective longitudinal section. Study population: 23 children from 4 to 7 years oldof both sexes, who were diagnosed with pulp necrosis; the information obtained was recorded in a data collection form. Results: A significant favorable difference was observed between clinical signs and symptoms at the beginning and at the end, after the application of the triclaritro antibiotic mixture during the experimental periods: 7 days and 15 days. Conclusion: It was concluded that the triclaritro antibiotic mixture is effective in $100 \%$ of the pulp treatments of deciduous teeth of children from 4 to 7 years old who attended the dental clinic of the Jorge Basadre Grohmann National University.
\end{abstract}

Keywords: Endodontics, pulpectomy, primary teeth, antibiotics. 


\section{Introducción}

En la actualidad, a pesar de las campañas sobre prevención y cuidado de la cavidad bucal en niños, aún se mantiene una alta prevalencia de caries dental (1-3), muchas de las cuales no reciben el tratamiento a tiempo haciendo que el diagnóstico de pulpitis reversible continúe hasta evolucionar a una degeneración pulpar, y que en muchos de los casos afecte a la zona periradicular del diente. Para poder salvaguardar la pieza dentro de la cavidad bucal, y evitar futuros problemas, el tratamiento indicado es la pulpectomía; que consiste en la eliminación completa del tejido pulpar, y su reemplazo con un material reabsorbible (4). La finalidad del tratamiento es conservar la pieza decidua en boca, y así poder mantener el espacio para la erupción del diente permanente; además, la presencia del diente deciduo también es importante para la fonación, crecimiento, desarrollo, respiración y estética del niño $(5,6)$.

La técnica endodóntica no instrumentada o llamada también terapia LSTR 3Mix-MP (Lesion Sterilization and Tissue Repair) consiste en la eliminación de la pulpa necrótica que se encuentra en la cámara pulpar y la colocación de mezclas antibióticas en la entrada de los conductos radiculares (6-8). Esta técnica es catalogada como una técnica mínimamente invasiva, de bajo costo y que permite reducir el tiempo de trabajo. Una de las mezclas más utilizadas es la pasta de Hoshino, usada en la terapia LSTR como 3Mix-MP (9-11), la cual tiene como efecto secundario la pigmentación marrón de la pieza decidua, ya que tiene entre sus componentes a la minociclina (12-16).

La mezcla antibiótica triclaritro es una modificación de la pasta de Hoshino; contiene: metronidazol, ciprofloxacino, claritromicina y propilenglicol dentro de sus componentes. La principal ventaja es que no ocasiona cambios cromáticos en la pieza dental, ofreciendo similares resultados en el postratamiento (17); por lo cual, este trabajo tuvo como propósito analizar la efectividad clínica del uso de la mezcla antibiótica triclaritro en piezas deciduas con diagnóstico de absceso apical agudo de 23 niños de 4 a 7 años con controles a los 7 y 15 días.

\section{Metodología}

El diseño de la investigación es descriptivo, de corte longitudinal, y prospectivo. Para la obtención de datos se elaboró un instrumento con la finalidad de registrar la evolución de las manifestaciones clínicas (dolor, rubor, tumor y movilidad dental). El primer control, después de la aplicación de la mezcla antibiótica triclaritro, se realizó a los 7 días; y el segundo control se realizó a los 15 días postratamiento. Terminada la recolección de la muestra se realizó una base de datos en el programa Excel 2018 para la evaluación estadística que consiste en la elaboración de tablas de contingencia y posterior representación gráfica con el uso de diagramas de barras.

Población: La población estuvo integrada por niños de 4 a 7 años con diagnóstico de absceso apical agudo, que acudieron a la clínica odontológica de la Universidad Nacional Jorge Basadre Grohmann, Tacna, entre los meses de agosto a noviembre del 2017.

Unidad de estudio: Dientes deciduos con diagnóstico de absceso apical agudo de 23 niños entre 4 a 7 años que se realizaron la técnica endodóntica no instrumentada con uso de la mezcla antibiótica triclaritro que cumplían con los criterios de inclusión y exclusión.

\section{Criterios de Inclusión:}

- Niños de ambos sexos, de 4 a 7 años de edad.

- Piezas dentales deciduas con diagnóstico de absceso apical agudo en piezas deciduas anteriores o posteriores.

- Piezas dentales deciduas que, al examen radiográfico, presenten un máximo de un tercio de reabsorción radicular.

- Niños cuyos padres y/o tutores hayan firmado el consentimiento informado.

- Niños que tengan dentición decidua o mixta.

\section{Criterios de exclusión:}

- Niños con enfermedades sistémicas.

- Niños que presenten dentición permanente.

- Piezas dentales deciduas que, al examen radiográfico, muestren imágenes radiopacas del diente con tratamiento pulpar coronal.

- Piezas dentales deciduas que, al examen clínico, presenten movilidad dentaria grado 3 .

- Piezas dentarias deciduas fracturadas a nivel de furca.

\section{Secuencia de preparación de la mezcla triclaritro}

Para la preparación de la mezcla antibiótica triclaritro, se empleó 1 tableta de cada antibiótico en sus presentaciones de $500 \mathrm{mg}$, cada una. Posteriormente, con el uso de una hoja de bisturí n. ${ }^{\circ} 15$, se removió la cubierta entérica de cada una de ellas y se procedió a triturar las drogas en un mortero. 


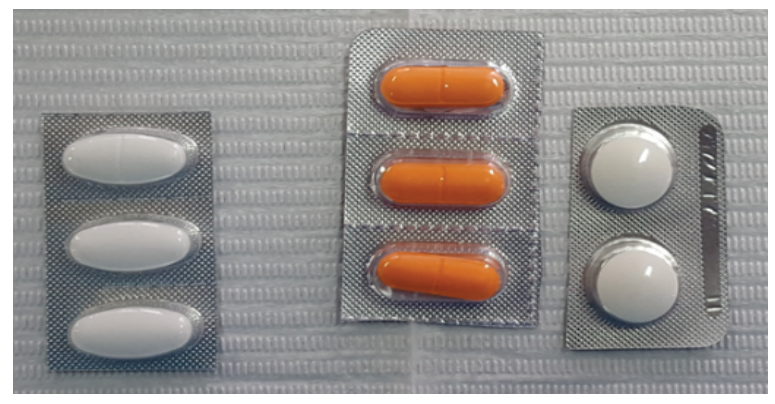

Figura 1. Metronidazol, ciprofloxacino y claritromicina.

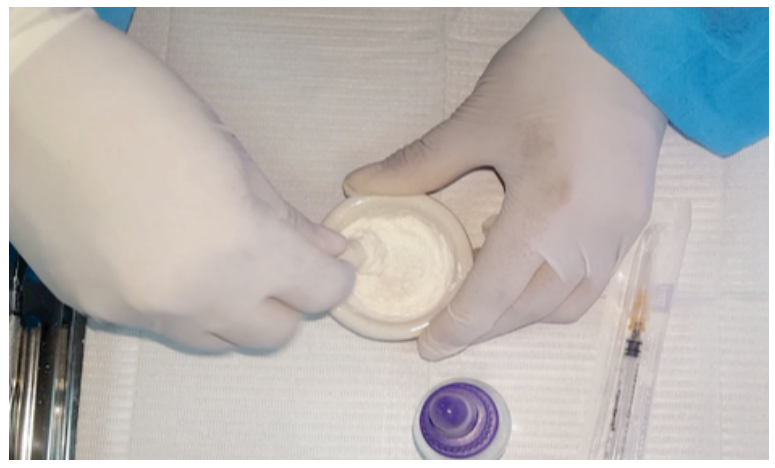

Figura 2. Triturado de las tabletas en mortero.

Luego se combinó con 20 gotas del vehículo (propilenglicol) hasta obtener la consistencia de pasta, y se transportó con una espátula a una jeringa hipodérmica para facilitar su manipulación.

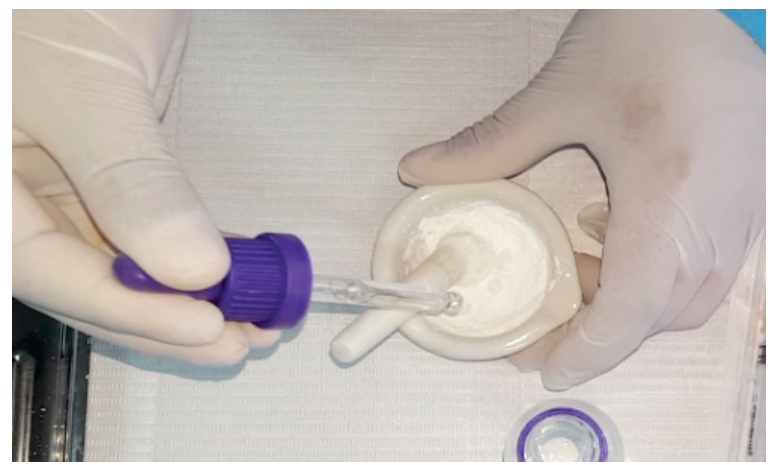

Figura 3. Adición de propilenglicol a la mezcla.

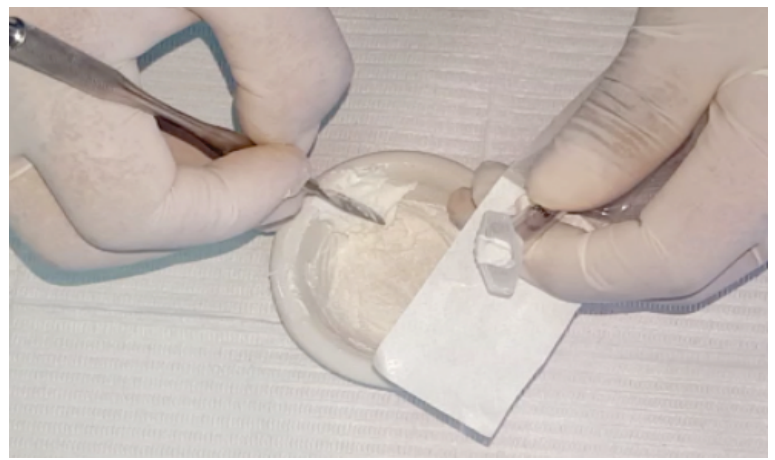

Figura 4. Transporte de la mezcla a una jeringa hipodérmica.

\section{Secuencia de tratamiento no instrumentado con la mezcla antibiótica triclaritro}

Para llevar a cabo la terapia LSTR 3Mix-MP con uso de la mezcla antibiótica triclaritro se realiza la toma de una radiografía antes del tratamiento; profilaxis de la pieza dental; aplicación de anestesia tópica para luego, en el caso de piezas superiores, proceder con la anestesia infiltrativa, y para piezas inferiores, anestesia troncular; aislamiento absoluto del campo operatorio; remoción del tejido cariado con fresas a alta velocidad o con curetas de dentina; apertura cameral y eliminación del tejido pulpar residual; conformación de pequeñas cavidades a la entrada de los conductos (18) que alojen la mezcla antibiótica triclaritro; irrigación con hipoclorito de sodio al $0.5 \%(6,11)$; colocación de la mezcla antibiótica triclaritro en las cavidades preparadas con ayuda de la jeringa hipodérmica (de no poderse realizar, se extiende la mezcla por el piso de la cámara pulpar); sellado de la cavidad con ionómero de vidrio fotocurable de restauración; control de la oclusión, y toma de una radiografía de control al finalizar el procedimiento.

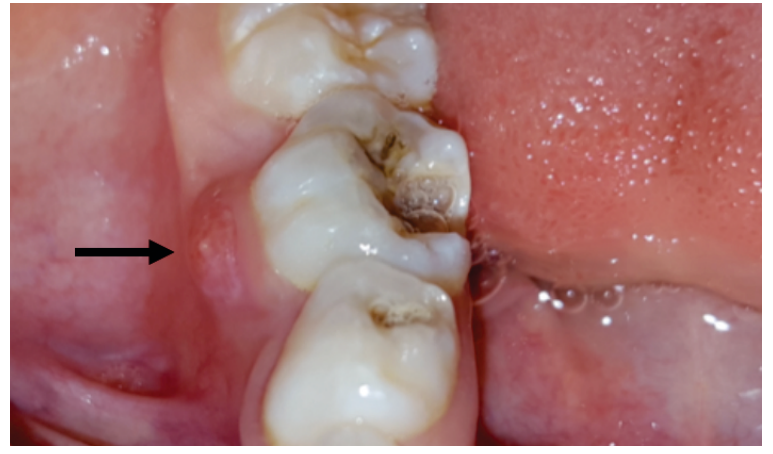

Figura 5. Pieza 8.5 necrosada con presencia de absceso.

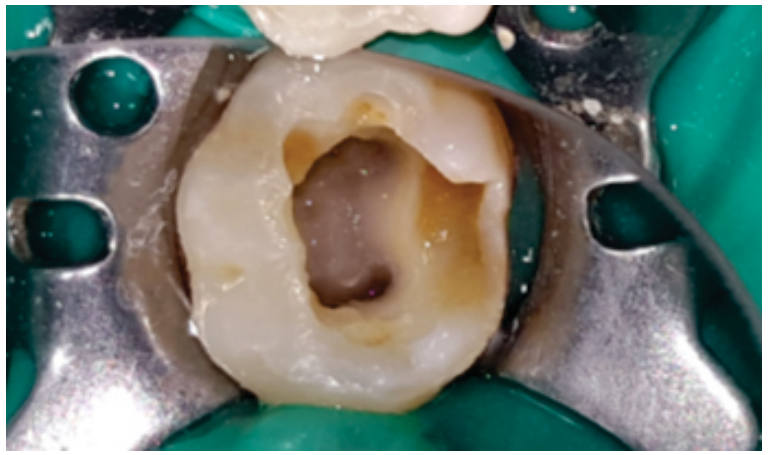

Figura 6. Apertura cameral. 


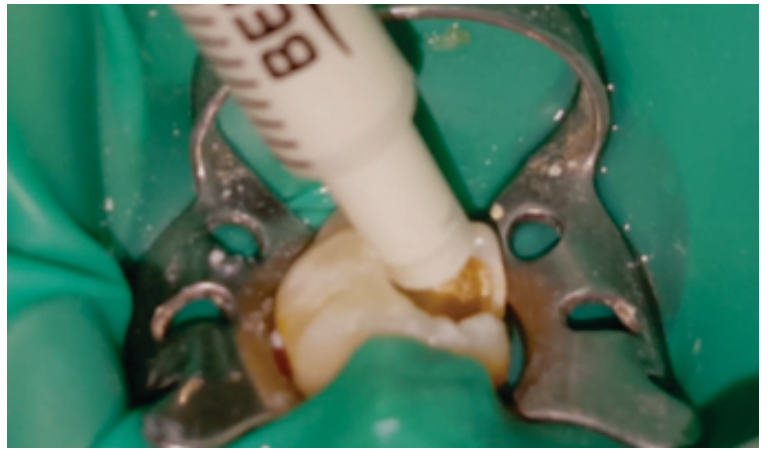

Figura 7. Aplicación de la mezcla antibiótica triclaritro.

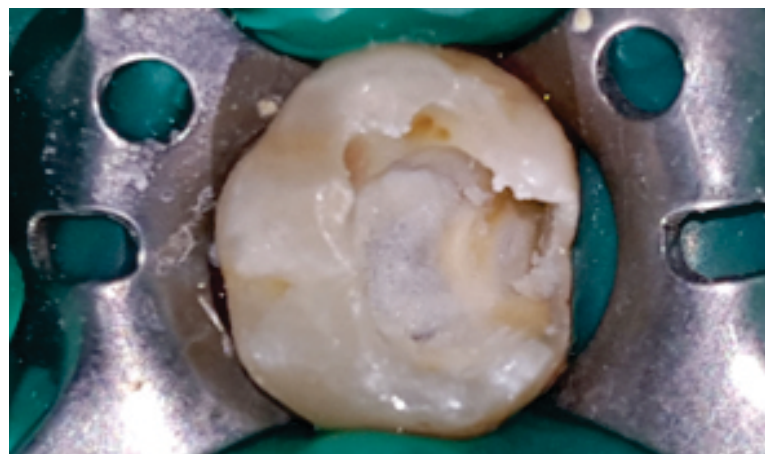

Figura 8. Mezcla antibiótica triclaritro en la entrada de los conductos.

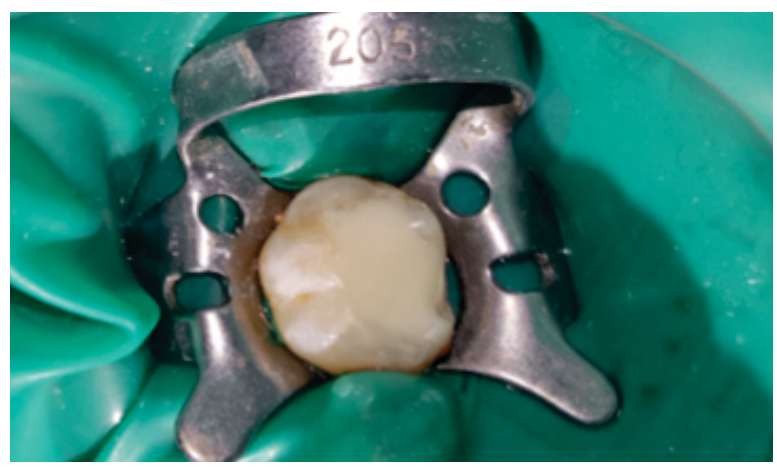

Figura 9. Restauración con ionómero de vidrio fotocurable de restauración.

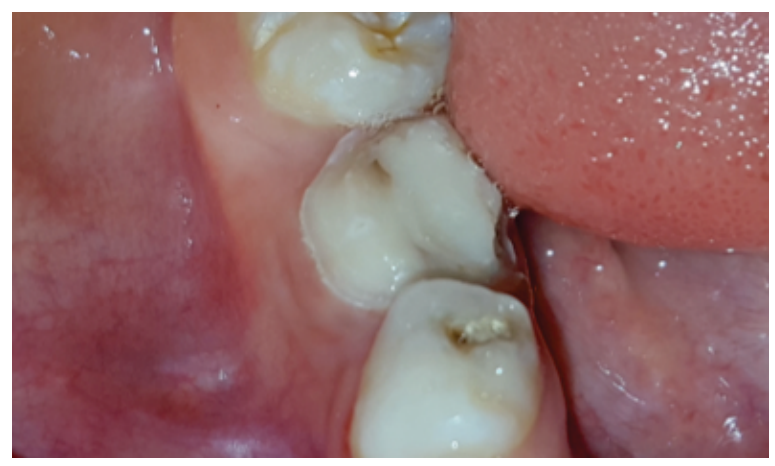

Figura 10. Evaluación a los 7 días; nótese ausencia del absceso.

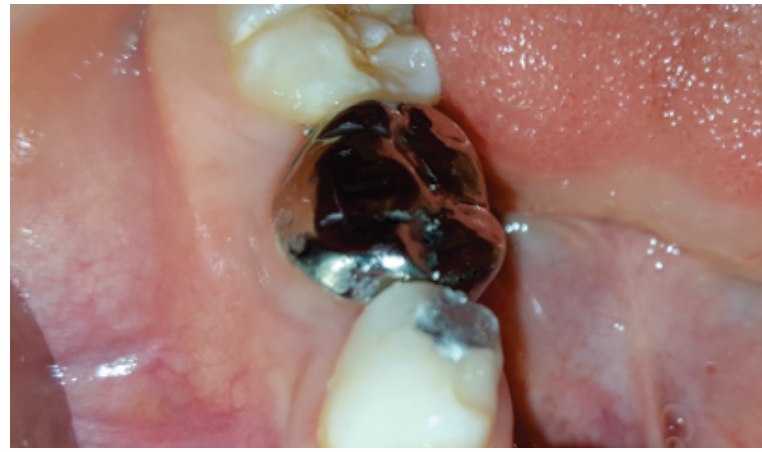

Figura 11. Evaluación a los 15 días; se optó por la cementación de una corona metálica debido a la gran pérdida de estructura dental.

\section{Radiografías}

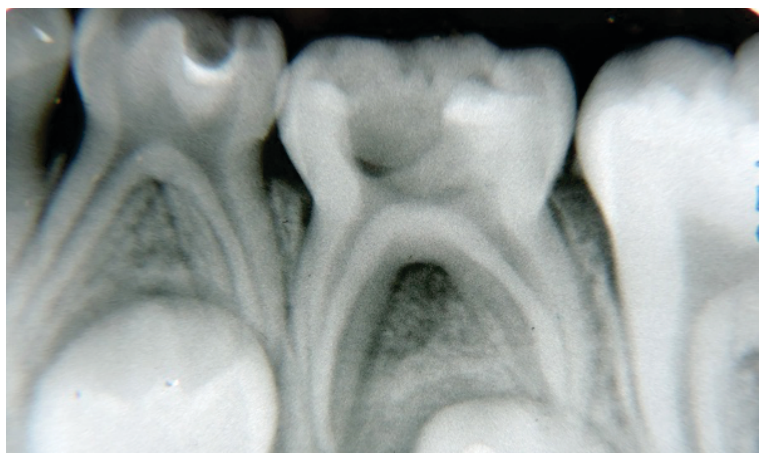

Figura 12. Radiografía inicial.

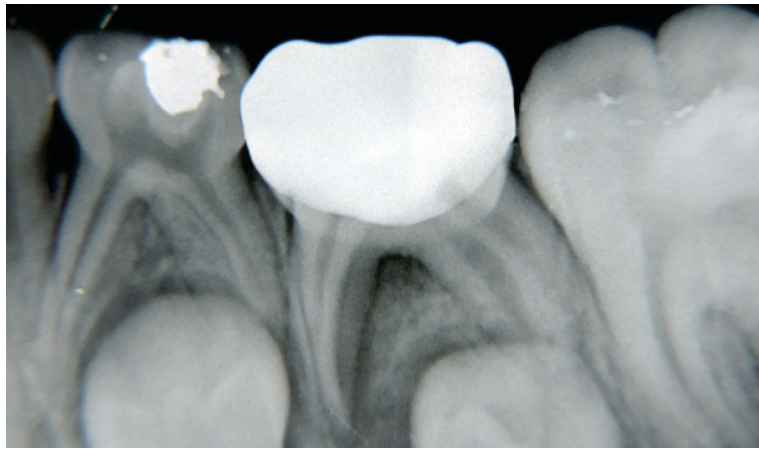

Figura 13. Radiografía final.

\section{Resultados}

Luego del procesamiento y análisis de datos correspondientes se obtuvieron los siguientes resultados: 
Cuadro 1. Remisión del dolor en tratamientos pulpares de dientes deciduos con diagnóstico de absceso apical agudo con la mezcla antibiótica triclaritro en niños de 4 a 7 años.

\begin{tabular}{|c|c|c|c|c|c|c|c|c|c|c|c|c|c|c|c|c|c|c|}
\hline & \multicolumn{6}{|c|}{ Dolor espontáneo } & \multicolumn{6}{|c|}{$\begin{array}{c}\text { Dolor provocado a la percusión } \\
\text { vertical }\end{array}$} & \multicolumn{6}{|c|}{$\begin{array}{c}\text { Dolor provocado a la percusión } \\
\text { horizontal }\end{array}$} \\
\hline & \multicolumn{2}{|c|}{ Inicial } & \multicolumn{2}{|c|}{7 días } & \multicolumn{2}{|c|}{15 días } & \multicolumn{2}{|c|}{ Inicial } & \multicolumn{2}{|c|}{7 días } & \multicolumn{2}{|c|}{15 días } & \multicolumn{2}{|c|}{ Inicial } & \multicolumn{2}{|c|}{7 dias } & \multicolumn{2}{|c|}{15 días } \\
\hline & $\mathrm{N}$ & $\%$ & $\mathrm{~N}$ & $\%$ & $\mathrm{~N}$ & $\%$ & $\mathrm{~N}$ & $\%$ & $\mathrm{~N}$ & $\%$ & $\mathrm{~N}$ & $\%$ & $\mathrm{~N}$ & $\%$ & $\mathrm{~N}$ & $\%$ & $\mathrm{~N}$ & $\%$ \\
\hline Si & 23 & 100,0 & 0 & 0 & 0 & 0 & 23 & 100,0 & 7 & 30,4 & 0 & 0 & 23 & 100,0 & 2 & 8,7 & 0 & 0 \\
\hline No & 0 & 0 & 23 & 100,0 & 23 & 100,0 & 0 & 0 & 16 & 69,6 & 23 & 100,0 & 0 & 0 & 21 & 91,3 & 23 & 100,0 \\
\hline Total & 23 & 100,0 & 23 & 100,0 & 23 & 100,0 & 23 & 100,0 & 23 & 100,0 & 23 & 100,0 & 23 & 100,0 & 23 & 100,0 & 23 & 100,0 \\
\hline
\end{tabular}

Los dientes deciduos con presencia de dolor espontáneo, provocado a la percusión vertical y horizontal, son del $100 \%$ al inicio del tratamiento, del total de niños, de los cuales pasado los 15 días del tratamiento se observó igualmente ausencia de dolor también en un $100 \%$.

Gráfico 1. Remisión del dolor en tratamientos pulpares de dientes deciduos con diagnóstico de absceso apical agudo con la mezcla antibiótica triclaritro en niños de 4 a 7 años.

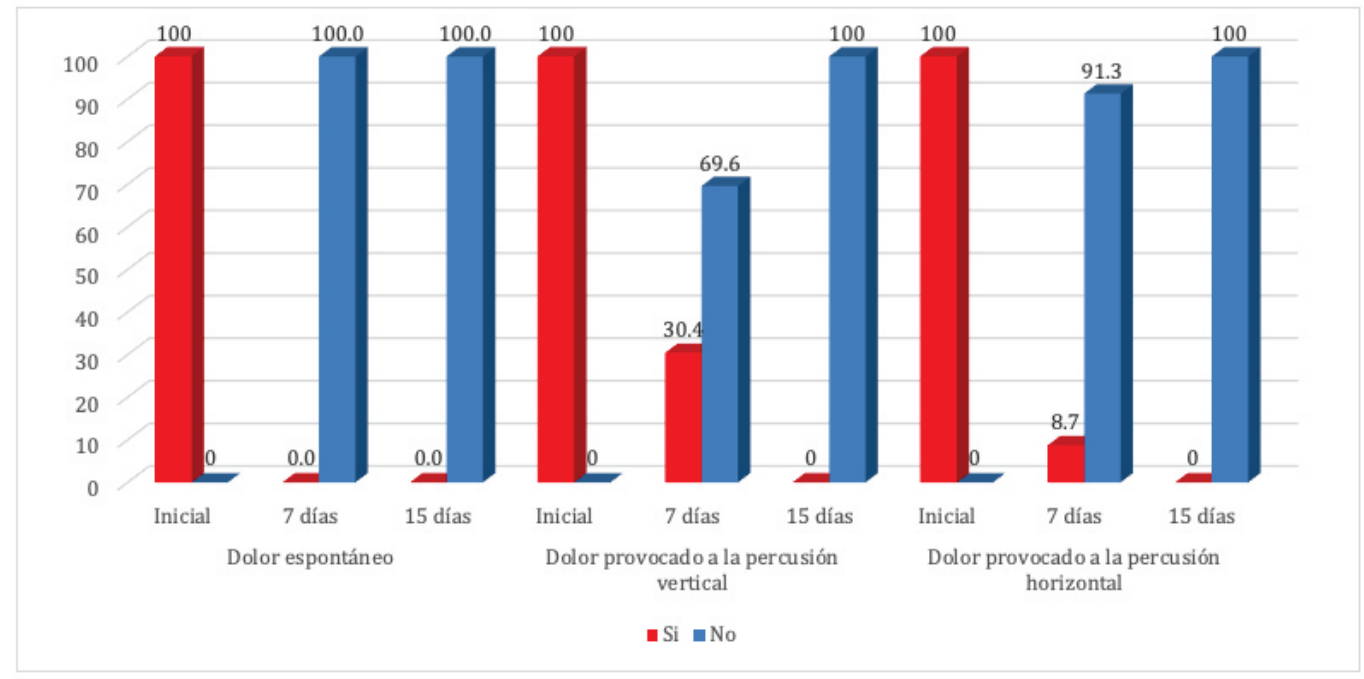

Cuadro 2. Remisión del rubor en tratamientos pulpares de dientes deciduos con diagnóstico de absceso apical agudo con la mezcla antibiótica triclaritro en niños de 4 a 7 años.

\begin{tabular}{l|c|c|c|c|c|c|}
\hline & \multicolumn{6}{|c}{ Rubor } \\
\hline & \multicolumn{2}{|c|}{ Inicial } & \multicolumn{2}{|c|}{7 días } & \multicolumn{2}{c}{15 días } \\
\hline Si & N & $\%$ & N & $\%$ & N & $\%$ \\
\hline No & 23 & 100,0 & 6 & 26,1 & 0 & 0 \\
\hline Total & 0 & 0 & 17 & 73,9 & 23 & 100,0 \\
\hline
\end{tabular}

Los dientes deciduos con presencia de tumor son de $100 \%$ al inicio del tratamiento. Observándose ausencia de tumor a los 7 y 15 días pasado el tratamiento.

Gráfico 3. Remisión del tumor en tratamientos pulpares de dientes deciduos con diagnóstico de absceso apical agudo con la mezcla antibiótica triclaritro en niños de 4 a 7 años.

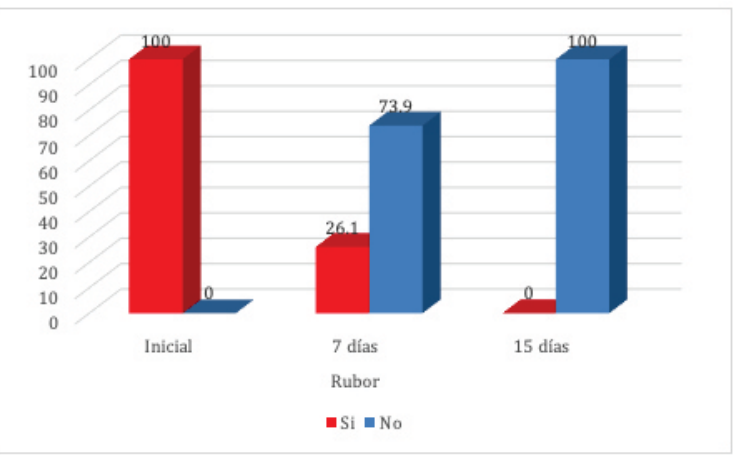

Fuente: Cuadro 3.

Cuadro 4. Disminución de la movilidad dentaria grado II en tratamientos pulpares de dientes deciduos con diagnóstico de absceso apical agudo con la mezcla antibiótica triclaritro en niños de 4 a 7 años. 


\begin{tabular}{l|c|c|c|c|c|c|} 
& \multicolumn{6}{|c}{ Tumor } \\
\hline & \multicolumn{2}{|c|}{ Inicial } & \multicolumn{2}{c}{7 días } & \multicolumn{2}{c}{15 días } \\
\hline & N & $\%$ & N & $\%$ & N & $\%$ \\
\hline Si & 23 & 100,0 & 0 & 0 & 0 & 0 \\
\hline No & 0 & 0 & 23 & 100,0 & 23 & 100,0 \\
\hline Total & 23 & 100,0 & 23 & 100,0 & 23 & 100,0 \\
\hline
\end{tabular}

Los dientes deciduos con movilidad dental grado II son de $100 \%$ al inicio del tratamiento, de los cuales a los 15 días se observó movilidad dental grado I de 100\%.

Gráfico 4. Disminución de la movilidad dentaria grado II en tratamientos pulpares de dientes deciduos con diagnóstico de absceso apical agudo con la mezcla antibiótica triclaritro en niños de 4 a 7 años.

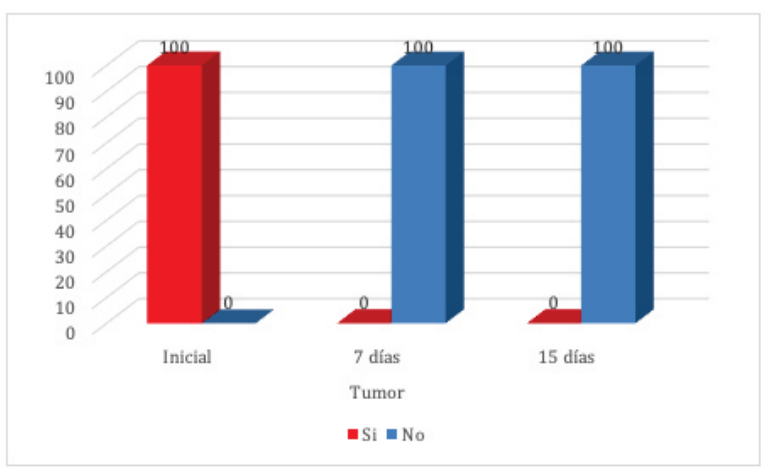

Fuente: Cuadro 4.

\section{Discusión}

La aplicación de mezclas antibióticas ha demostrado buenos resultados clínicos en dientes deciduos necróticos, e incluso es considerado como un procedimiento regenerativo de endodoncia (11). En este estudio, después de la aplicación de la mezcla antibiótica triclaritro, se observó resultados favorables en cuanto a las manifestaciones clínicas postratamiento, dando así una opción más conservadora y rápida para la resolución de casos diagnosticados con absceso apical agudo.

Mandras N. et al. (Italia-2013) (19) en su estudio in vitro demuestran que la aplicación de la mezcla antibiótica triclaritro evidencia una actividad bactericida de $89 \%$, esto es de 3 a 4 veces significativamente mayor en comparación con otras mezclas antibióticas de dicho estudio, estos datos concuerdan con los resultados obtenidos en nuestro estudio debido a la remisión de signos y síntomas clínicos postratamiento con la mezcla antibiótica triclaritro; así mismo, en cuanto a la decoloración, no se observan cambios de color en las piezas dentarias.

Denisova T. et al. (Italia-2013) (17) determinaron que el uso de la mezcla antibiótica triclaritro demuestra una eficacia bactericida mayor contra diferentes especies bacterianas tomadas de conductos radiculares infectados, en comparación con las mezclas trimix, bimix y trifosfo; esto se pudo comprobar en nuestro estudio debido a que se presentaron mejoras clínicas de signos y síntomas postratamiento.

Genta E. et al. (Italia-2016) (20), en su investigación, que se realizó en raíces dentales con presencia de Enterococcus faecalis, demostraron que la aplicación de la mezcla antibiótica triclaritro y trimix no tienen diferencias significativas en cuanto a la penetración en los túbulos de dentina, esto podría relacionarse con los resultados obtenidos en nuestro estudio, para lo cual utilizamos propilenglicol como vehículo debido a sus mejores propiedades (21); esto se pudo observar en la disminución de la sintomatología postratamiento.

Además, según las consultas realizadas en diferentes bases de datos de trabajos de investigación tales como PubMed, ScienceDirect, Alicia, Concytec y Renati; se obtiene como resultado, que existen trabajos de investigación como los de Takushige E. et al. (Japón2004) (18), Palacios M. (Ecuador-2015) (22), Quintana del Solar C. et al. (Perú-2012) (23) y Luna R. et al. (Perú-2016) (24), donde se estudia la efectividad de la mezcla antibiótica 3Mix-MP, la cual demostró ser efectiva para los casos de absceso apical agudo. La principal diferencia de la mezcla antibiótica triclaritro con la 3 Mix-MP, radica en un componente farmacológico dentro de su composición; puesto que la mezcla 3Mix-MP contiene minociclina, la cual es remplazada por claritromicina en la mezcla triclaritro; este cambio es realizado con la finalidad de poder eliminar el efecto de pigmentación postratamiento $(25,26)$. El intercambio de este componente farmacológico supone que existe una diferencia en cuanto a la acción que esta tuviera, y por lo tanto los resultados podrían tener una variación postratamiento después de la aplicación de dichas mezclas. Esto no se pudo comprobar en el presente estudio debido a que solo se utilizó la mezcla antibiótica triclaritro. No se observaron cambios radiográficos con la aplicación de la mezcla triclaritro.

\section{Conclusiones}

La mezcla antibiótica triclaritro es efectiva en el 100\% de los casos en la remisión de signos y síntomas, después del tratamiento pulpar de dientes deciduos diagnosticados con acceso apical agudo en niños de 4 a 7 años que acudieron a la Clínica Odontológica de la Universidad Nacional Jorge Basadre Grohmann (Tacna, 2017).

Se observó remisión de dolor, rubor y tumor en tratamientos pulpares de dientes deciduos con diagnóstico de absceso apical agudo con la mezcla antibiótica triclaritro de niños de 4 a 7 años en un 100\% de los casos, a los 15 días. Asimismo, hubo disminución 
de movilidad dentaria grado II en tratamientos pulpares de dientes deciduos con diagnóstico de absceso apical agudo con la mezcla antibiótica triclaritro en un $100 \%$ de los casos, a los 15 días.

Se recomienda realizar estudios con el uso de la mezcla antibiótica triclaritro en una población mayor para obtener resultados con un mínimo margen de error, lo que nos llevaría a encontrar una nueva opción terapéutica contra los casos diagnosticados con absceso apical agudo en dientes deciduos; también se recomienda realizar estudios longitudinales donde se considere obtener datos radiográficos para poder evaluar la eficacia en tiempos mayores a la presente investigación.

\section{Referencias bibliográficas}

1. Ministerio de Salud. Prevalencia nacional de caries dental, fluorosis del esmalte y urgencia de tratamiento en escolares de 6 a 8, 10, 12 y 15 años, Perú 2001 - 2002. [Internet]. Lima: Oficina General de Epidemiología; 2005. D i s p o n i b l e e n : http://www.dge.gob.pe/publicaciones/pub_ca ries/prevalencia_caries.pdf

2. Ministerio de Salud. Guía de práctica clínica para la prevención, diagnóstico y tratamiento de la caries dental en niños y niñas. Lima: Oficina General de Epidemiología; 2017.

3. Federación Dental Internacional. El desafío de las enfermedades bucodentales [Internet]. Brighton; 2015. Disponible en: https://www.fdiworlddental.org/sites/default/ files/media/documents/book_spreads_oh2_s panish.pdf

4. Sociedad Española de Odontopediatria. Protocolo para los tratamientos pulpares en dentición temporal [Internet]. Barcelona; 2019 . D i s p o n i b le e n: https://www.odontologiapediatrica.com/prot ocolos/pulpa/

5. Lillo OC, Quesada JRB. Endodoncia en la dentición temporal. En: Sahli CC, Aguadé EB, editors. Endodoncias técnicas clínicas y bases científicas. 3rd ed. Barcelona: Elsevier; 2014. p. 271-81.

6. Perona G, Mungi S. Tratamiento Endodóntico no Instrumentado en dientes deciduos. Rev odontopediatr latinoam [Internet]. $2014 ; 4(1): 53-64$. Disponible en: https://www.revistaodontopediatria.org/edici ones/2014/1/art-6/

7. Takushige T, Cruz EV, Moral AA, Hoshino E. Non-surgical treatment of pulpitis, including those with history of spontaneous pain, using a combination of antibacterial drugs. J LSTR Ther [Internet]. 2008; 7:1-5. Disponible en: http://1str.jp/e/_userdata/Takushige-
SavePulp-J LSTR.pdf

8. Nanda R, Koul M, Srivastava S, Upadhyay V, Dwivedi R. Clinical evaluation of 3 Mix and Other Mix in non-instrumental endodontic treatment of necrosed primary teeth. J Oral Biol Craniofac Res [Internet]. 2014 May;4(2):114-9. Disponible en: https://linkinghub.elsevier.com/retrieve/pii/S 2212426814000372

9. Sato I, Ando-Kurihara N, Kota K, Iwaku M, Hoshino E. Sterilization of infected root-canal dentine by topical application of a mixture of ciprofloxacin, metronidazole and minocycline in situ. Int Endod J [Internet]. 1996 Mar;29(2):118-24. Disponible en: http://www.ncbi.nlm.nih.gov/pubmed/92064 35

10. Hoshino E, Kurihara-Ando N, Sato I, Uematsu $\mathrm{H}$, Sato M, Kota K, et al. In-vitro antibacterial susceptibility of bacteria taken from infected root dentine to a mixture of ciprofloxacin, metronidazole and minocycline. Int Endod $\mathbf{J}$ [Internet]. 1996 Mar;29(2):125-30. D i s p o i b l e e n : http://doi.wiley.com/10.1111/j.13652591.1996.tb01173.x

11. Kontakiotis EG, Filippatos CG, Tzanetakis GN, Agrafioti A. Regenerative Endodontic Therapy: A Data Analysis of Clinical Protocols. J Endod [Internet]. 2015 Feb;41(2):146-54. Disponible en: https://linkinghub.elsevier.com/retrieve/pii/S 0099239914007857

12. Sato T, Hoshino E, Uematsu H, Noda T. In vitro antimicrobial susceptibility to combinations of drugs of bacteria from carious and endodontic lesions of human deciduous teeth. Oral Microbiol Immunol [Internet]. 1993 Jun;8(3):172-6. A Disponible en: http://doi.wiley.com/10.1111/j.1399302X.1993.tb00661.x

13. Lenherr P, Allgayer N, Weiger R, Filippi A, Attin T, Krastl G. Tooth discoloration induced by endodontic materials: a laboratory study. Int Endod J [Internet]. 2012 Oct;45(10):942-9. Disponible en: 
http://doi.wiley.com/10.1111/j.13652591.2012.02053.x

14. Akcay M, Arslan H, Topcuoglu HS, Tuncay O. Effect of calcium hydroxide and double and triple antibiotic pastes on the bond strength of epoxy resin-based sealer to root canal dentin. J Endod [Internet]. 2014 Oct;40(10):1663-7. D i s p o n b l e e n : http://www.ncbi.nlm.nih.gov/pubmed/2526074 1

15. Quispe-Salcedo A, Ida-Yonemochi H, Ohshima $\mathrm{H}$. Effects of a Triple Antibiotic Solution on Pulpal Dynamics after Intentionally Delayed Tooth Replantation in Mice. J Endod [Internet]. 2014 Oct;40(10):1566-72. Disponible en: https://linkinghub.elsevier.com/retrieve/pii/S00 99239914004518

16. Kahler B, Rossi-Fedele G. A Review of Tooth Discoloration after Regenerative Endodontic Therapy. J Endod [Internet]. 2016 Apr;42(4):563-9. Disponible en: https://linkinghub.elsevier.com/retrieve/pii/S00 99239915011528

17. Denisova T, Pasqualini D, Mandras N, Roana J, Allizond V, Crosasso P, et al. Valutazione dell'efficacia battericida e dell'incidenza di discromia di associazioni di antibiotici nella terapia di rivascolarizzazione. G Ital Endod [Internet]. 2013 Jun;27(1):45-9. Disponible en: https://linkinghub.elsevier.com/retrieve/pii/S1 1 21417113000125

18. Takushige T, Cruz EV, Moral AA, Hoshino E. Endodontic treatment of primary teeth using a combination of antibacterial drugs. Int Endod $\mathbf{J}$ [Internet]. 2004 Feb;37(2):132-8. Disponible e http://www.ncbi.nlm.nih.gov/pubmed/1487118 0

19. Mandras N, Roana J, Allizond V, Pasqualini D, Crosasso P, Burlando M, et al. Antibacterial efficacy and drug-induced tooth discolouration of antibiotic combinations for endodontic regenerative procedures. Int $\mathrm{J}$ Immunopathol Pharmacol [Internet]. 2013;26(2):557-63. D i s p o n i b l e e $\mathrm{n}$ : http://www.ncbi.nlm.nih.gov/pubmed/2375577 4

20. Genta E, Alovisi M, Cuffini AM, Mandras N, Luganini A, Roana J, et al. Confocal scanner laser evaluation of bactericidal effect of different antibiotic mixtures used for dental pulp regeneration: a preliminary study. G Ital Endod [Internet]. 2016 Jun;30(1):41-5. Disponible en: https://linkinghub.elsevier.com/retrieve/pii/S1 1 21417116300097

21. Cruz E V, Kota K, Huque J, Iwaku M, Hoshino E. Penetration of propylene glycol into dentine. Int Endod J [Internet]. 2002 Apr;35(4):330-6. D i s p o i b l e e n : http://www.ncbi.nlm.nih.gov/pubmed/1205993 3

22. Palacios MKM. Evaluación de la eficacia del uso de la pasta triantibiótica como material de obturación en piezas deciduas con necrosis pulpar, en la Clínica de Odontopediatría. Facultad Piloto de Odontología Periodo Lectivo 2014-2015. Universidad de Guayaquil; 2015.

23. Quintana del Solar CI, Quispe La Rosa M. Efectividad de una pasta tri-antibiótica en pieza decidua necrótica con absceso periapical y fístula. Odontol Sanmarquina [Internet]. 2012 May 14;15(2):31-4. Disponible en: http://revistasinvestigacion.unmsm.edu.pe/inde x.php/odont/article/view/2041

24. Luna RDA, Trujillo AHB. Efectividad de la pasta Hoshino o Trimix-MP versus ZOE empleadas en la cicatrización apical de piezas dentales con necrosis pulpar en niños de 3 a 7 años de edad, Lima - 2015. Universidad Nacional Hermilio Valdizán;2016.

25. Kim J-H, Kim Y, Shin S-J, Park J-W, Jung I-Y. Tooth Discoloration of Immature Permanent Incisor Associated with Triple Antibiotic Therapy: A Case Report. J Endod [Internet]. 2010 Jun;36(6):1086-91. Disponible en: https://linkinghub.elsevier.com/retrieve/pii/S00 99239910002803

26. Bak S-Y, Kim Y-J, Kim J-W, Jan K-T, Lee S-H, Kim C-C, et al. Treatment of tooth discoloration associated with triple antibiotic therapy: case reports. J Korean Acad Pediatr Dent [Internet]. 2012 Feb 29;39(1):43-50. Disponible en: http://koreascience.or.kr/journal/view.jsp?kj=S OCGB@\&py=2012\&vnc=v39n1\&sp=43
Correspondencia:

jram.lpb@gmail.com
Fecha de recepción : 31 de marzo de 2020

Fecha de aceptación : 01 de junio de 2020 